\title{
Revisiting McKinsey's 'Syntactical' Construction of Modality
}

\author{
M.J. Cresswell \\ Victoria University of Wellington \\ Wellington New Zealand \\ max.cresswell@vuw.ac.nz
}

\begin{abstract}
The present paper looks at how to produce a completeness proof for J.C.C. McKinsey's syntactical account of modal logic, by comparing McKinsey 'models' with the current approach using frames. It also looks at the motivation behind the system which we now call S4M; and uses this motivation to produce a McKinsey semantics for that system.
\end{abstract}

In McKinsey 1945, J.C.C. McKinsey produced what he called a 'syntactical' account of modal logic, which predated the now standard possible worlds semantics. ${ }^{1}$ On p. 83 McKinsey says:

As the intuitive basis for the syntactical definition of possibility, I take the position that to say a sentence is possible means that there exists a true sentence of the same form. Thus, for example, it would be said that the sentence, 'Lions are indigenous to Alaska,' is possible, because of the fact that the sentence, 'Lions are indigenous to Africa' has the same form and is true. ${ }^{2}$

McKinsey immediately recognises that this needs to be made precise, and announces the following way of dealing with the problem:

\footnotetext{
${ }^{1}$ For a more general account of the history of modal logic see Goldblatt 2006. It is an honour to be able to contribute to the celebration of Rob's achievements in this issue of the Australasian Journal of Logic.

${ }^{2}$ One can see why he calls his account 'syntactical', and why he speaks of a sentence as possible; but its use of the notion of truth makes it equally accurate to call it a 'semantics', and that is how I will frequently think of it in this paper. A discussion of McKinsey's semantics may be found on pp. 165-169 of Humberstone 2015.
}

Australasian Journal of Logic (17:2) 2020, Article no. 3 
I shall avoid these difficulties by supposing merely that we are given a certain set of substitutions which take the sentences of a certain language into other (or sometimes the same) sentences of the language; and I shall call a sentence possible, if some substitution of the set takes it into a true sentence.

McKinsey is not specific about what kind of language this is, though his motivation might suggest that he thinks of it as a regimentation of a natural language whose sentences have definite truth values. The regimentation would consist in using 'constants' to represent the 'descriptive' symbols like 'Alaska' and 'Africa' in his motivating example, in contrast with the 'logical' symbols which determine the 'logical form' of the sentence in question. This of course is in line with Quine 1934 who takes them to express sentences of a particular natural language whose sentences have definite truth values. It is perhaps significant that the language introduced on p. 84 contains no formation rules, and it is not clear just what its atomic sentences are. Luckily however it is possible to present McKinsey's account in a language $\mathcal{L}$ of modal propositional logic, and that is the procedure adopted in this paper. $^{3}$

\section{McKinsey Models}

I will base my discussion on a langauge with a primitive necessity operator $\square$. McKinsey actually follows Lewis in using $\diamond$ as a possibility operator and expressing necessity by $\sim \diamond \sim$, so to avoid confusion with McKinsey's languages I will call the language of the present paper $\mathcal{L}$. Assume then a language $\mathcal{L}$ of propositional modal logic, based on the 'falsum' $\perp$ and the operators $\supset$ and $\square$, with the usual definitions of the other operators - in particular with the possibility operator $\diamond$ defined so that $\diamond \alpha=\mathrm{df} \quad \sim \square \sim \alpha$. Let the atomic wff of $\mathcal{L}$ be $\perp$ and the members of a set $\mathrm{P}$ of propositional variables $p_{1}, p_{2}, \ldots, p_{n}$ and so on. The complex wff are made up from the atomic wff in the usual way. $\mathcal{L}$ can be interpreted by what we shall call McKinsey-models. Adapting McKinsey's definitions from p. 84 - supplemented by his addition of a modal operator on p. 85 - to a language based on $\perp, \supset$ and $\square$, his account can be presented as follows. In addition to a truth-value assignment $\mathrm{M}$ - which assigns exactly one of two truth values to the atomic wff - assume that $\Sigma$ is a non-empty set of substitutions, where

\footnotetext{
${ }^{3} \mathrm{~A}$ description of McKinsey's own procedure and terminology is found in the appendix to the present paper. $(\S 7)$
}

Australasian Journal of Logic (17:2) 2020, Article no. 3 
Each $s \in \Sigma$ is a function which associates with every $p \in \mathrm{P}$ a PC-wff (i.e. a non-modal wff) $\alpha$.

The extension of the substitutions to cover all PC wff in $\mathcal{L}$ can be defined in the following way. For every substitution $s$ :

$$
\begin{aligned}
& \mathbf{S} \perp s(\perp)=\perp \\
& \mathbf{S} \supset s(\alpha \supset \beta)=(s(\alpha) \supset s(\beta))
\end{aligned}
$$

Then, on p. 85, Mckinsey extends the language to a modal language which he calls $L_{1}$ and it is his $L_{1}$ which corresponds to our $\mathcal{L}$. Substitutions in modal wff are defined by:

$$
\mathbf{S} \square s(\square \alpha)=\square s(\alpha)^{4}
$$

It is vital to appreciate that although $s(\alpha)$ can be a modal formula, since $\alpha$ itself may contain modal operators, $s(\alpha)$ does not introduce any modal operators not already in $\alpha$. A McKinsey model for $\mathcal{L}$ is a pair $\langle\mathrm{M}, \Sigma\rangle$ where $\mathrm{M}$ is a truth-value assignment to the members of $\mathrm{P}$, and $\Sigma$ a non-empty set of substitutions satisfying (1). I write $\langle\mathrm{M}, \Sigma\rangle \mid=\alpha$ to indicate that $\alpha$ is true in $\langle\{\mathrm{M}, \Sigma\rangle$, and $\langle\mathrm{M}, \Sigma\rangle=\alpha$ otherwise. $|=$ can be defined for all wff of $\mathcal{L}$ by the rules

$$
\begin{aligned}
& \text { For any } p \in \mathrm{P},\langle\mathrm{M}, \Sigma\rangle \mid=p \text { iff } \mathrm{M} \text { gives } p \text { the value true. } \\
& \langle\mathrm{M}, \Sigma\rangle=\perp \\
& \langle\mathrm{M}, \Sigma\rangle \mid=\alpha \supset \beta \text { unless }\langle\mathrm{M}, \Sigma\rangle \mid=\alpha \text { and }\langle\mathrm{M}, \Sigma\rangle=\beta \text {. } \\
& \langle\mathrm{M}, \Sigma\rangle \mid=\square \alpha \text { iff }\langle\mathrm{M}, \Sigma\rangle \mid=s(\alpha) \text { for every } s \in \Sigma \text {. }
\end{aligned}
$$

McKinsey's definition on p. 84 requires that $s(p)$, for any $p \in \mathrm{P}$, be a non-modal wff, and it is easy to justify a prohibition on allowing $s(p)$ to be a modal formula. For suppose that $\Sigma=\left\{s_{1}, s_{2}\right\}$, where $s_{1}(p)=p$ and $s_{2}(p)=\sim \square p$, and that $\mathrm{M}$ assigns the value true to $p$. Then, $\langle\mathrm{M}, \Sigma\rangle \mid=p$, and by (5), $\langle\mathrm{M}, \Sigma\rangle \mid=\square p$ iff, for, every $s \in \Sigma,\langle\mathrm{M}, \Sigma\rangle \mid=p$ and $\langle\mathrm{M}, \Sigma\rangle \mid=\sim \square p$; and clearly there can be no such model. By restricting $s(p)$ to PC wff we can be sure that in evaluating $\square \alpha, s(\alpha)$, for every $s \in \Sigma$, will be of lower modal degree than $\square \alpha$, and may be assumed to have already been given a value. (This is so even when $\alpha$ contains modal operators, for, since $s$ substitutes only PC wff for its atomic wff $\alpha$ 's modal degree will not be raised.)

\footnotetext{
${ }^{4}$ Allowing for his different primitives $\mathbf{S} \perp$ and $\mathbf{S} \supset$ correspond to McKinsey's $A_{1}$ and $A_{2}$ on p. $84\left(C_{2}\right.$ and $C_{3}$ on p. 85). $\mathrm{S} \square$ corresponds to $C_{1}$ on p. 85.
}

Australasian Journal of Logic (17:2) 2020, Article no. 3 


\section{Equivalence of models}

Assume the definition of truth in a (Kripke) model $\langle\mathrm{W}, \mathrm{R}, \mathrm{V}\rangle$, as found say, in Hughes and Cresswell 1996. Our task is to produce, for any denumerable Kripke model, a McKinsey model which, in a sense to be defined, satisfies the same formulae. Because McKinsey's semantics is expressed in terms of substitutions of formulae in a denumerable language it is necessary in this construction to have a denumerable Kripke model. The aim of this section is to prove that for every denumerable Kripke model $\langle W, R, V\rangle$ in which $R$ is serial - that is, in which for every $w \in \mathrm{W}$ there is some $w^{\prime} \in \mathrm{W}$ such that $w \mathrm{R} w w^{\prime}$ - there is a McKinsey model $\left\langle\mathrm{M}_{\mathrm{V}}, \Sigma_{\mathrm{R}}\right\rangle$ which is equivalent to $\langle\mathrm{W}, \mathrm{R}, \mathrm{V}\rangle$.

Let $\mathrm{F}_{\mathrm{R}}$ be the set of all functions $f$ from $\mathrm{W}$ into $\mathrm{W}$ such that $f \subseteq \mathrm{R}$. Then

$$
w \mathrm{R} w^{\prime} \text { iff for some } f \in \mathrm{F}_{\mathrm{R}}, f(w)=w^{\prime} .
$$

That $\mathrm{R}$ is serial is required to ensure that $f(w)$ is defined for every $w \in \mathrm{W} .^{5}$ The key idea in the McKinsey model to be constructed is that, in place of the truth of a variable $p$ in a world $w$, we use the truth of a separate but corresponding variable $p_{\mathrm{w}}$. Where $\langle\mathrm{W}, \mathrm{R}, \mathrm{V}\rangle$ is any model, let the set $\Sigma_{\mathrm{R}}-$ strictly $\Sigma_{\langle W, R, V\rangle}$ - be the set of substitutions based on $\langle\mathrm{W}, \mathrm{R}, \mathrm{V}\rangle$. Assume, for each $w \in \mathrm{W}$, a disjoint copy of $\mathrm{P}$. That is to say, $\mathrm{P}_{w}=\left\{p_{w}: p \in \mathrm{P}\right\}$, ensuring that, for $w \neq w \prime, \mathrm{P}_{w} \cap \mathrm{P}_{w^{\prime}}=\varnothing$. From this it follows that if $p_{w}=p_{w^{\prime}}$ then $w=w^{\prime}$, Let $\mathrm{P}_{\omega}$ be the union of all the $\mathrm{P}_{w} \mathrm{~s}$, and let $\mathcal{L}_{\omega}$ be the modal propositional language based on $\mathrm{P}_{\omega}$.

For $f \in \mathrm{F}_{\mathrm{R}}$ let $s_{\mathrm{f}}$ denote the substitution such that, for every $p \in \mathrm{P}$ and every $w \in \mathrm{W}, s_{\mathrm{f}}\left(p_{\mathrm{W}}\right)=p_{f(w)}$

Now define $\Sigma_{\mathrm{R}}$ as

$$
\left\{s: \exists f\left(f \in \mathrm{F}_{\mathrm{R}} \wedge s=s_{\mathrm{f}}\right\} .\right.
$$

From (7) and (8) we may conclude that $\Sigma_{R}$ has two properties. The first is:

For any $w$ and $w^{\prime}$ such that $w \mathrm{R} w^{\prime}$, there is some $s \in \Sigma_{\mathrm{R}}$ such that, for every $p \in \mathrm{P}, s\left(p_{\mathrm{w}}\right)=p_{w^{\prime}}$

\footnotetext{
${ }^{5}$ The theorems which follow can be extended to models in which $\mathrm{R}$ is not serial, but we then need to allow substitutions which are undefined for some variables. That possibility is ruled out by requiring that $\Sigma$ be non-empty and that every $s \in \Sigma$ is defined for every $p \in \mathrm{P}$. McKinsey only considers logics which contain $p \supset \diamond p$, and therefore $\square p \supset \diamond p$ as theorems, and so adapting his definitions to allow undefined substitutions, though possible, not only introduces complexities, but departs somewhat from his intentions.
}

Australasian Journal of Logic (17:2) 2020, Article no. 3 
since if $w \mathrm{R} w$, there is some $f \in \mathrm{F}_{\mathrm{R}}$ such that $f(w)=w$. Let $s$ be $s_{\mathrm{f}}$. Then, by (7), $s_{\mathrm{f}}\left(p_{\mathrm{w}}\right)=p_{f(w)}$ for every $p \in \mathrm{P}$, and by (8) $s \in \Sigma_{\mathrm{R}}$. The second property is that for every $w$ and $w^{\prime} \in \mathrm{W}$ :

$$
\text { For any } s \in \Sigma \text {, if } s\left(p_{\mathrm{w}}\right)=p_{w^{\prime}} \text { for any } p \in \mathrm{P} \text {, then } w \mathrm{R} w w^{\prime} \text {. }
$$

For suppose that $s\left(p_{\mathrm{w}}\right)=p_{w}$ for some $p \in \mathrm{P}$. Since $s \in \Sigma_{\mathrm{R}}$ then $s$ will be $s_{\mathrm{f}}$ for some $f \in \mathrm{F}_{\mathrm{R}}$, where, for every $q \in \mathrm{P}, s_{\mathrm{f}}\left(q_{\mathrm{w}}\right)=q_{f(w)}$ and $w \mathrm{R} f(w)$. In particular $s_{\mathrm{f}}\left(p_{\mathrm{w}}\right)=p_{f(w)}$, and so $p_{f(w)}=p_{w^{\prime}}$. So $w^{\prime}=f(w)$, and therefore $w \mathrm{R} w^{\prime}$.

For any wff $\alpha$ in $\mathcal{L}$ let $\alpha_{w}$ (in $\mathcal{L}_{\omega}$ ) be $\alpha$ with $p_{w}$ replacing $p$ for every variable $p$ in $\alpha$.

It is trivial that:

$$
\perp_{w}=\perp,(\alpha \supset \beta)_{w}=\left(\alpha_{w} \supset \beta_{w}\right) \text { and }(\square \alpha)_{w}=\square \alpha_{w} .
$$

From (9) and (12) we have that, for any wff $\alpha$

For any $w$ and $w^{\prime}$ such that $w \mathrm{R} w w^{\prime}$, there is some $s \in \Sigma_{\mathrm{R}}$ such that $s\left(\alpha_{\mathrm{w}}\right)=\alpha_{w^{\prime}}$

and from (10) and (12) we have that, for any wff $\alpha$

$$
\text { If } s\left(\alpha_{w}\right)=\alpha_{w^{\prime}} \text { for any wff } \alpha \text { in } \mathcal{L} \in \mathrm{P} \text {, then } w \mathrm{R} w^{\prime} .
$$

For $p \in \mathrm{P}$ and $w \in \mathrm{W}$, let $\mathrm{M}_{\mathrm{V}}$ be an assignment giving $p_{\mathrm{W}}$ the value true if $\mathrm{V}(p, w)=1$ and false otherwise. Then $\left\langle\mathrm{M}_{\mathrm{V}}, \Sigma_{\mathrm{R}}\right\rangle$ is a McKinsey model for $\mathcal{L}_{\omega}$ satisfying the condition

$$
\left\langle\mathrm{M}_{\mathrm{V}}, \Sigma_{\mathrm{R}}\right\rangle \mid=p_{\mathrm{w}} \text { iff } \mathrm{V}(p, w)=1 .
$$

THEOREM 1 For any wff $\alpha$ in $\mathcal{L},\left\langle\mathrm{M}_{\mathrm{V}}, \Sigma_{\mathrm{R}}\right\rangle \mid=\alpha_{w}$ iff $\mathrm{V}(\alpha, w)=1$.

Proof. The result is defined to hold for the variables, clearly holds for $\perp$ and is preserved by $\supset$. For $\square$, suppose first that $\mathrm{V}(\square \alpha, w)=0$. Then there is some $w^{\prime}$ such that $w \mathrm{R} w w^{\prime}$ and $\mathrm{V}\left(\alpha, w^{\prime}\right)=0$. So by the induction hypothesis $\left\langle\mathrm{M}_{\mathrm{V}}, \Sigma_{\mathrm{R}}\right\rangle \Rightarrow \alpha_{w^{\prime}}$. But by (13) there is some $s \in \Sigma$ such that $s\left(\alpha_{w}\right)=\alpha_{w^{\prime}}$, and therefore $\left\langle\mathrm{M}_{\mathrm{V}}, \Sigma_{\mathrm{R}}\right\rangle \Rightarrow \square \alpha_{w}$, i.e., $\left\langle\mathrm{M}_{\mathrm{V}}, \Sigma_{\mathrm{R}}\right\rangle=(\square \alpha)_{w}$. Now suppose that $\left\langle\mathrm{M}_{\mathrm{V}}, \Sigma_{\mathrm{R}}\right\rangle=(\square \alpha)_{w}$. Then $\left\langle\mathrm{M}_{\mathrm{V}}, \Sigma_{\mathrm{R}}\right\rangle \Rightarrow \square \alpha_{w}$, and so for some $s \in \Sigma,\left\langle\mathrm{M}_{\mathrm{V}}, \Sigma_{\mathrm{R}}\right\rangle=$ $s\left(\alpha_{w}\right)$. Now $s\left(\alpha_{w}\right)$ is $\alpha_{w}$, for some $w^{\prime}$, and so, by the induction hypothesis, $\mathrm{V}\left(\alpha, w^{\prime}\right)=0$, and since, by (14), wR $w$ ' then $\mathrm{V}(\square \alpha, w)=0$.

Australasian Journal of Logic (17:2) 2020, Article no. 3 
It is worth noting that, in the McKinsey model $\left\langle\mathrm{M}_{\mathrm{V}}, \Sigma_{\mathrm{R}}\right\rangle$ produced in this construction, for any variable $p$, and any $s \in \Sigma_{\mathrm{R}}, s(p)$ is also a variable. This means that, for the purposes of theorem 1 we could have imposed an even more restrictive condition on $s\left(p_{\mathrm{w}}\right)$ than that it be a PC-wff. It need only be a variable. However we shall follow McKinsey in defining models to allow $s(p)$ to be any PC wff.

\section{Characterisation Classes}

The purpose of this section is to use a class of denumerable models which characterise a normal system of propositional modal logic in order to define a corresponding class of McKinsey models which also characterise that system. ${ }^{6}$ Assume the standard definition of a normal system of propositional modal logic. $\mathrm{S}$ is normal iff it contains all tautologies of the classical propositional calculus (PC), the modal formula

K $\quad \square(p \supset q) \supset(\square p \supset \square q)$

and is closed under the rules of uniform substitution, modus ponens and necessitation. Where $\vdash_{\mathrm{S}} \alpha$ indicates that $\alpha$ is a theorem of $\mathrm{S}$ the three transformation rules may be written as:

US If $\vdash_{\mathrm{s}} \alpha$, then $\vdash_{\mathrm{s}} \beta$ where $\beta$ is obtained from $\alpha$ by uniform substitution of wff for its propositional variables.

MP If $\vdash_{\mathrm{s}} \alpha$ and $\vdash_{\mathrm{s}} \alpha \supset \beta$ then $\vdash_{\mathrm{s}} \beta$.

$\mathbf{N} \quad$ If $\vdash_{\mathrm{s}} \alpha$ then $\vdash_{\mathrm{s}} \square \alpha$.

In this paper we shall restrict consideration to systems which contain D, that is to say, normal systems containing the formula

D $\square p \supset \diamond p$.

We now prove a theorem about all normal modal systems which corresponds to the well-known canonical model theorem of Kripke semantics. The models we have in mind cannot be the canonical model for a system $S$ since, in a canonical model there have to be as many worlds as there are maximal S-consistent sets of wff, and W would therefore be non-denumerable.

\footnotetext{
${ }^{6}$ It is important here to realise that we are talking about characterisation by a class of models not by a class of frames. There may be a notion of frame appropriate to a McKinsey model, but it is not obvious what it is. The set $\Sigma$ of substitutions in such a model is certainly independent of the value assignment $M$, but it is still essentially tied to particular variables in a way in which a relational frame is not.
}

Australasian Journal of Logic (17:2) 2020, Article no. 3 
But a canonical model is more than we need, since all we require is that any S-consistent set $\Lambda$ of wff can be simultaneously satisfied in a world in a denumerable model; and techniques like the 'subordination' method found in Chapter 7 of Hughes and Cresswell 1984 shew how to construct a denumerable model of the appropriate kind. ${ }^{7}$ From this it follows that every normal modal system $S$ is characterised by what we may call a characterisation class $\mathcal{E}_{\mathrm{S}}$ of denumerable models whereby

$$
\begin{aligned}
& \text { If } \vdash_{\mathrm{S}} \alpha \text { then } \mathrm{V}(\alpha, w)=1 \text { for every } w \in \mathrm{W} \text { where }\langle\mathrm{V}, \mathrm{R}, \mathrm{V}\rangle \\
& \in \mathcal{E}_{\mathrm{S}} \\
& \text { If } \dashv_{\mathrm{S}} \alpha \text { then there is some }\langle\mathrm{V}, \mathrm{R}, \mathrm{V}\rangle \in \mathcal{E}_{\mathrm{S}} \text { such that for some } \\
& w \in \mathrm{W} \mathrm{V}(\alpha, w)=0 .
\end{aligned}
$$

We now let $\mathcal{E}_{S}^{\prime}$ be the set of McKinsey models which correspond to the models in $\mathcal{E}_{S}$ in the way defined in section 2 . Then, by theorem 1 we have:

$$
\begin{aligned}
\text { THEOREM } 2 & \text { If } \vdash_{\mathrm{S}} \alpha \text { then }\left\langle\mathrm{M}_{\mathrm{V}}, \Sigma_{\mathrm{R}}\right\rangle \mid=\alpha_{\mathrm{w}} \text { for every }\left\langle\mathrm{M}_{\mathrm{V}}, \Sigma_{\mathrm{R}}\right\rangle \in \mathcal{E}_{\mathrm{S}}^{\prime} \\
& \text { If } \dashv_{\mathrm{S}} \alpha \text { then there is some }\left\langle\mathrm{M}_{\mathrm{V}}, \Sigma_{\mathrm{R}}\right\rangle \in \mathcal{E}_{\mathrm{S}}^{\prime} \text { such that } \\
& \left\langle\mathrm{M}_{\mathrm{V}}, \Sigma_{\mathrm{R}}\right\rangle=\alpha_{\mathrm{w}} .
\end{aligned}
$$

That is to say, $\mathcal{E}_{\mathrm{S}}^{\prime}$ characterises $\mathrm{S}$ if $\mathcal{E}_{\mathrm{S}}$ does.

\section{Completeness}

The principal task of this section is to prove the completeness of S4 with respect to two conditions McKinsey imposes on sets of substitutions. Although completeness results for McKinsey's semantics have been obtained in Drake 1962, Drake's algebraic proofs rely on the finite model property of the systems in question, and thus will not generalise to all extensions of D. ${ }^{8}$ His article predates the emergence of the canonical model method for completeness based on Kripke-style possible world models, which, as we have seen, allows a characterisation result in terms of McKinsey models to be obtained for all extensions of $\mathrm{D}$.

Given a model $\langle\mathrm{M}, \Sigma\rangle$, call a wff $\alpha\langle\mathrm{M}, \Sigma\rangle$-true iff $\langle\mathrm{M}, \Sigma\rangle \mid=\alpha$, when is evaluated according to (5), and call $\alpha\langle\mathrm{M}, \Sigma\rangle$-valid iff $\beta$ is $\langle\mathrm{M}, \Sigma\rangle$-true for

\footnotetext{
${ }^{7}$ See also Makinson 1966.

${ }^{8}$ In fact his 'substitutions' turn out to be operators in a finite algebra, and seem better understood as operators on sets of worlds rather than substitutions in McKinsey's sense. He then connects these algebras with the modal deductive systems by means of a tableau completeness proof.
}

Australasian Journal of Logic (17:2) 2020, Article no. 3 
every $\beta$ obtained from $\alpha$ by uniform substitution for $\alpha$ 's variables. ${ }^{9}$ Say that $\alpha$ is $\langle\mathrm{M}, \Sigma\rangle$-equivalent to $\beta$ iff $\alpha \equiv \beta$ is $\langle\mathrm{M}, \Sigma\rangle$-valid. Something like this may be what McKinsey has in mind:

The provable formulas of the usual systems are, presumably, to be taken as those which become true when the sentential variables are replaced by arbitrary constant sentences. (p. 85)

The distinction between truth and validity in a McKinsey model is that soundness cannot be proved with respect to truth. Although $\langle\mathrm{M}, \Sigma\rangle$-truth is preserved by modus ponens it is not preserved either by uniform substitution or necessitation. For consider a model $\langle\mathrm{M}, \Sigma\rangle$ in which a variable, say $p$, is assigned the value true, while another variable, say $q$, is assigned false, and in which there is some $s \in \Sigma$ such that $s(p)=q$. It is easy to see that in this case $\langle\mathrm{M}, \Sigma\rangle \mid=p$ but $\langle\mathrm{M}, \Sigma\rangle \Rightarrow q$ and $\langle\mathrm{M}, \Sigma\rangle=\square p$.

We first shew that the system $D$ is sound with respect to the class of all McKinsey models. It is sufficient to establish that the axioms of $\mathrm{D}$ are $\langle\mathrm{M}, \Sigma\rangle$-valid in every model $\langle\mathrm{M}, \Sigma\rangle$; and that the transformation rules preserve $\langle\mathrm{M}, \Sigma\rangle$-validity in every $\langle\mathrm{M}, \Sigma\rangle$. Obviously $\langle\mathrm{M}, \Sigma\rangle \mid=\beta$ for every instance $\beta$ of a valid PC wff $\alpha$. The only modal axioms in D are $\mathbf{K}, \square(p \supset q)$ $\supset(\square p \supset \square q)$ and D.

Lemma $3 \quad \mathbf{K}$ is $\langle\mathrm{M}, \Sigma\rangle$-valid for every model.

Proof. It is sufficient to prove that $\square(\alpha \supset \beta) \supset(\square \alpha \supset \square \beta)$ is $\langle\mathrm{M}, \Sigma\rangle$-true for every $\alpha$ and $\beta$. Suppose $\langle\mathrm{M}, \Sigma\rangle=\square(\alpha \supset \beta) \supset(\square \alpha \supset \square \beta)$. Then we have (i) $\langle\mathrm{M}, \Sigma\rangle \mid=\square(\alpha \supset \beta)$, (ii) $\langle\mathrm{M}, \Sigma\rangle \mid=\square \alpha$, but (iii) $\langle\mathrm{M}, \Sigma\rangle=\square \beta$. From (iii) there is some $s \in \Sigma$ such that $\langle\mathrm{M}, \Sigma\rangle \mid=s(\beta)$. But, from (ii) by (5). $\langle\mathrm{M}, \Sigma\rangle s(\alpha)$, and then by $(4),\langle\mathrm{M}, \Sigma\rangle \Rightarrow s(\alpha) \supset s(\beta)$. But $(s(\alpha) \supset s(\beta))=s(\alpha \supset \beta)$, and so $\langle\mathrm{M}, \Sigma\rangle \Rightarrow s(\alpha \supset \beta)$, contradicting (i).

Lemma $4 \quad \mathrm{D}$ is $\langle\mathrm{M}, \Sigma\rangle$-valid for every model.

Proof. We shew that for any wff $\alpha, \square \alpha \supset \diamond \alpha$ is $\langle\mathrm{M}, \Sigma\rangle$-true. Suppose not, then for some $\langle\mathrm{M}, \Sigma\rangle$. (i) $\langle\mathrm{M}, \Sigma\rangle \mid=\square \alpha$ and (ii) $\langle\mathrm{M}, \Sigma\rangle=\diamond \alpha$. Let $s$ be any member of $\Sigma$. Since $\Sigma$ is non-empty then there will be some $s \in \Sigma$. From (i) $\langle\mathrm{M}, \Sigma\rangle \mid=s(\alpha)$, and from (ii) $\langle\mathrm{M}, \Sigma\rangle=\mid s(\alpha)$, which is a contradiction.

\footnotetext{
${ }^{9} \beta$ here may be a modal formula, unlike the values of the substitutions for variables, licensed by $\Sigma$. $\langle\mathrm{M}, \Sigma\rangle$-validity comes close to the 'Carnap-validity' defined on p. 58 of Cresswell 2013, though is of course importantly different from it.
} 
We now note that the transformation rules preserve $\langle\mathrm{M}, \Sigma\rangle$-validity in any model $\langle\mathrm{M}, \Sigma\rangle$. For US we merely note that if every instance of $\alpha$ is true in $\langle\mathrm{M}, \Sigma\rangle$, and $\beta$ is an instance of $\alpha$ then every instance of $\beta$ is also true in $\langle\mathrm{M}, \Sigma\rangle$. For necessitation suppose that some instance of $\square \alpha$ is not true in a model $\langle\mathrm{M}, \Sigma\rangle$, i.e. that $\langle\mathrm{M}, \Sigma\rangle \Rightarrow \square \beta$ where $\beta$ is an instance of $\alpha$. Then there is some $s \in \Sigma$ such that $\langle\mathrm{M}, \Sigma\rangle=s(\beta)$, and so $s(\beta)$ is not $\langle\mathrm{M}, \Sigma\rangle$-true. But $s(\beta)$ is an instance of $\beta$, and therefore an instance of $\alpha$, which is to say that $\alpha$ is not $\langle\mathrm{M}, \Sigma\rangle$-valid, contrary to the hypothesis. Where $\mathrm{S}$ is a normal modal system axiomatised by a set $\mathrm{Ax}_{\mathrm{S}}$ of wff we can say that a wff $\alpha$ is S-valid iff it is $\langle\mathrm{M}, \Sigma\rangle$-valid in every $\langle\mathrm{M}, \Sigma\rangle$ in which all the members of $A x_{S}$ are $\langle\mathrm{M}, \Sigma\rangle$-valid. The argument just given ensures that every $\alpha$ which can be derived from $\mathrm{Ax}_{\mathrm{S}}$ by US, MP and Nec is S-valid:

Theorem 5 Suppose that $S$ is a normal modal system defined by a collection $A x_{S}$ of axioms, and all members of $\mathrm{Ax}_{\mathrm{S}}$ are $\langle\mathrm{M}, \Sigma\rangle$-valid. Then if $\vdash_{\mathrm{S}} \alpha, \alpha$ is also $\langle\mathrm{M}, \Sigma\rangle$-valid.

What theorem 5 means is that in order to prove the soundness, in terms of $\langle\mathrm{M}, \Sigma\rangle$-validity, of any extension of $\mathrm{D}$ in terms of a set of McKinsey models, it is sufficient to establish the validity of its proper axioms in that set of models. And that of course means establishing that where $\alpha$ is one of these axioms then any substitution instance of $\alpha$ (including instances where modal wff are substituted for $\alpha$ 's variables) is true in each one of these models. By analogy with the possible-worlds semantics you can study particular systems by imposing conditions on $\Sigma$. Two conditions on substitutions which McKinsey imposes are:

Ref There is some $s \in \Sigma$ such that for every wff $\alpha, s(\alpha)=\alpha$.

Trans For any $s$ and $s /$ in $\Sigma$ there is some $s^{\prime \prime} \in \Sigma$ such that for all wff $\alpha$, $\left.s^{\prime \prime}(\alpha)\right)=\mathrm{s}\left(\mathrm{s}^{\prime}(\alpha)\right){ }^{10}$

It is not difficult to see that these are intuitively plausible conditions to impose on substitutions. McKinsey proves that these two conditions in conjunction with 5 give a system at least as strong as $\mathrm{S} 4$. The proofs of the $\langle\mathrm{M}, \Sigma\rangle$-validity of $\mathbf{T}(\square p \supset p)$ given Ref and of $\mathbf{4}(\square p \supset \square \square p)$ given Trans are trivial variants of the proofs found on p. 90 of McKinsey's article. To obtain S5 one adds the following condition:

Sym For any $s \in \sum$ there is some $s^{\prime} \in \sum$ such that $s^{\prime}(s(\alpha))=\alpha$ for all wff $\alpha$.

With Ref and Sym alone you get the soundness of the system B, which is T

\footnotetext{
${ }^{10}$ Ref and Trans are McKinsey's $A_{3}$ and $A_{4}$ on p. 84, where he calls the $s$ in Ref, $s_{1}$. The $s, s \prime$, and $s^{\prime \prime}$ in Trans are called $s_{\mathrm{n}}, s_{\mathrm{m}}$ and $s_{\mathrm{t}}$. Note that by $\mathbf{S} \perp-\mathbf{S} \square$, provided Ref and Trans hold for the variables, they will hold for every formula.
}

Australasian Journal of Logic (17:2) 2020, Article no. 3 
together with the axiom B ( $p \supset \square \sim \square p)$. S5 is S4 + B. Sym is not intuitively plausible for substitutions. For while it makes sense to obtain $p \supset p$ from $p$ $\supset q$ by substitution $p$ for $q$, it does not make sense to think of obtaining $p \supset$ $q$ by substituting $q$ for $p$ in $p \supset p$. There are of course substitutions which do satisfy Sym. An example would occur if we restricted the substitution rule so that distinct variables must be replaced by distinct variables, since it is easy to see that this construal validates $\mathbf{B} .{ }^{11}$

We now use theorem 1 to prove the completeness of $S 4$ with respect to McKinsey models.

Theorem 6 If a wff $\alpha$ is $\langle\mathrm{M}, \Sigma\rangle$-valid in every model $\langle\mathrm{M}, \Sigma\rangle$ which satisfies Ref and Trans then $\vdash_{\mathrm{S}_{4}} \alpha$.

Proof. We know that $\mathrm{S} 4$ is characterised by a class $\mathcal{E}_{S 4}$ of denumerable Kripke models in which $\mathrm{R}$ is reflexive and transitive, and therefore we know, from theorem 1, that there is also a corresponding class $\mathcal{E}_{S 4}^{\prime}$ of McKinsey models which characterises $\mathrm{S} 4$. It therefore suffices to shew that since $\langle\mathrm{W}, \mathrm{R}, \mathrm{V}\rangle$ in $\mathcal{E}_{S 4}$ is reflexive and transitive then the corresponding $\left\langle\mathrm{M}_{V}, \Sigma_{R}\right\rangle$ obtained from $\langle\mathrm{W}, \mathrm{R}, \mathrm{V}\rangle$ by the method of section 2 satisfies Ref and Trans.

From the definition of $\Sigma_{\mathrm{R}}$ in (8) we know that for any $w$ and $w^{\prime}$ such that $w \mathrm{R} w^{\prime}$, there is some $s \in \Sigma_{\mathrm{R}}$ such that, for every $p \in \mathrm{P}$,

$$
s\left(p_{\mathrm{w}}\right)=p_{w^{\prime}}
$$

and since $\mathrm{R}$ is reflexive in S4 this means that for every $w \in \mathrm{W}$ there is some $s \in \Sigma_{\mathrm{R}}$ such that, for every $p \in \mathrm{P}$,

$$
s\left(p_{\mathrm{w}}\right)=p_{w} .
$$

Although that is not quite sufficient to shew that there is a single $s \in \Sigma_{\mathrm{R}}$ for which 19 holds, we can, nevertheless, augment $\Sigma_{\mathrm{R}}$ by adding an additional $s^{*}$ which satisfies 19 for every $w \in \mathrm{W}$. The only change to the proof of theorem 1 is to note that (14) still applies when $\Sigma_{\mathrm{R}}$ is replaced by $\Sigma_{\mathrm{R}} \cup\left\{s^{*}\right\}$, and that it is sufficient to shew that for any $w \in \mathrm{W}$, if $s^{*}\left(p_{\mathrm{W}}\right)=p_{w^{\prime}}$ then $w \mathrm{R} w^{\prime}$. But if $s^{*}(w)=w^{\prime}$ then $w=w^{\prime}$, and in that case, since $\langle\mathrm{W}, \mathrm{R}, \mathrm{V}\rangle$ is a model for $\mathrm{S} 4, \mathrm{R}$

\footnotetext{
${ }^{11}$ That is, $\mathbf{B}$ is $\langle\mathrm{M}, \Sigma\rangle$-valid when substitutions are restricted to distinct variables for distinct variables. This may be proved as follows. Suppose that $\langle\mathrm{M}, \Sigma\rangle=\square \sim \square \alpha$. Then there will be a wff $\alpha^{\prime}$ obtainable from $\alpha$ by replacement of distinct $p_{1}, \ldots, p_{\mathrm{n}}$ in $\alpha$ by $q_{1}, \ldots, q_{\mathrm{n}}$ (where, for $1 \leqslant \mathrm{i} \leqslant \mathrm{n}$ and $1 \leqslant \mathrm{j} \leqslant \mathrm{n}, p_{\mathrm{i}}$ may be the same as $q_{\mathrm{j}}$ but $p_{\mathrm{i}} \neq p_{\mathrm{j}}$ and $q_{\mathrm{i}} \neq q_{\mathrm{j}}$ for $\mathrm{i} \neq \mathrm{j}$ ) such that $\langle\mathrm{M}, \Sigma\rangle=\mid \square \alpha^{\prime}$. So $\langle\mathrm{M}, \Sigma\rangle \mid=\square \alpha$ and so $\langle\mathrm{M}, \Sigma\rangle \mid=\beta$, where $\beta$ is any wff obtained from $\alpha^{\prime}$ by any replacement of distinct variables. One of these replacements will be that of $q_{\mathrm{i}}$ by $p_{\mathrm{i}}$, and in that case $\beta$ is $\alpha$, and so $\langle\mathrm{M}, \Sigma\rangle \mid=\alpha$, and therefore $\langle\mathrm{M}, \Sigma\rangle=\sim \alpha$.
}

Australasian Journal of Logic (17:2) 2020, Article no. 3 
is reflexive and therefore $w \mathrm{R} w$.

To satisfy Trans we need to require that for any $s_{1}$ and $s_{2} \in \Sigma_{\mathrm{R}}$ we can add an $s_{3}$ to $\Sigma_{\mathrm{R}}$ with the property that, for every $w \in \mathrm{W}$, and every $p \in$ $\mathrm{P}, s_{3}\left(p_{\mathrm{w}}\right)=s_{1}\left(s_{2}\left(p_{\mathrm{w}}\right)\right)$. So suppose that there are $s_{1}$ and $s_{2}$ such that, where $w \mathrm{R} w w^{\prime}$ and $w^{\prime} \mathrm{R} w^{\prime \prime}$,

$$
\text { (i) } s_{1}\left(p_{\mathrm{w}}\right)=p_{w^{\prime}} \text {, and } s_{2}\left(p_{w^{\prime}}\right)=p_{w^{\prime \prime}} \text {. }
$$

If we add to $\Sigma_{\mathrm{R}}$ an $s_{3}$ such that

$$
\text { (ii) } s_{3}\left(p_{\mathrm{w}}\right)=p_{w^{\prime \prime}}
$$

all we need is that $w \mathrm{R} w^{\prime \prime}$, and this is guaranteed by the transitivity of R.

\section{S4M}

In section 2 of his paper (pp. 91-93) McKinsey uses an example to shew that his semantics does not satisfy S5. This is because $\sim(1=2)$ is possible, since it is true. But it is not necessarily possible since when 1 is substituted for 2 we obtain a falsehood, because $\sim(1=1)$ is false. McKinsey notes, on p. 92, that the substitution used in this example, which he calls $s_{2}$ has the property that where $\alpha$ is any sentence and $s_{\mathrm{n}}$ any substitution the formula

$$
s_{\mathrm{n}}\left[s_{2}(\alpha)\right] \equiv s_{2}(\alpha)
$$

is valid, which validates a wff he calls $(F)$, which, in our notation is:

$$
\mathrm{F} \quad(\square \diamond p \wedge \square \diamond q) \supset \diamond(p \wedge q)
$$

It is known that in the presence $\mathrm{S} 4, \mathbf{F}$ is derivable from

$$
\text { M } \quad \square \diamond p \supset \diamond \square p .
$$

$\mathbf{M}$ is nowadays called the McKinsey Axiom and S4 + M is called S4M. ${ }^{12}$ In Kripke frames for S4M we add a 'finality' condition ensuring that each world can see a world which can only see itself. (See Hughes and Cresswell 1996, p. 131). This corresponds to a condition that McKinsey discusses on p. 92:

There is some $s_{2} \in \Sigma$ such that, for every $s \in \Sigma$ and every wff $\alpha, s\left(s_{2}(\alpha)\right)=s_{2}(\alpha)$.

\footnotetext{
${ }^{12}$ the name $\mathbf{M}$ is due to Lemmon and Scott 1977, p. 74. McKinsey 1945 p. 92, called S4M, S4.1, but, as he notes on p. 93, it is not contained in S5, and is therefore today not normally referred to as S4.1. Sobocinski 1964 p. 77 called the system K1, and it is discussed on pp. 131-134 of Hughes and Cresswell 1996, where a derivation is provided of F from M.
}

Australasian Journal of Logic (17:2) 2020, Article no. 3 
While that may enable a completeness proof it does not really highlight what it is about $s_{2}$ which makes it satisfy 20 - that where you have a substitution which turns a wff into a tautology or a contradiction then no further substitution can change the truth value of that formula. In a sense that is what happens in final worlds, and also what happens in any formula made up entirely from logical constants. Call a wff of $\mathcal{L}$ a constant wff iff its only atomic formulae are $T$ and $\perp$, where $T$ may be defined as $\perp \supset \perp$.

LEMMA 7 If $\alpha$ is a constant wff then either $\alpha$ is $\langle\mathrm{M}, \Sigma\rangle$-equivalent to $T$ or $\alpha$ is $\langle\mathrm{M}, \Sigma\rangle$-equivalent to $\perp$.

Proof. It is sufficient to shew that either $\langle\mathrm{M}, \Sigma\rangle \mid=\alpha$ for all $\langle\mathrm{M}, \Sigma\rangle$, or else $\langle\mathrm{M}, \Sigma\rangle=\alpha$ for all $\langle\mathrm{M}, \Sigma\rangle$, since, in the former case $\alpha$ will be equivalent to $\top$ and in the latter case to $\perp$. Since, for any $\langle\mathrm{M}, \Sigma\rangle$ either $\langle\mathrm{M}, \Sigma\rangle \mid=\alpha$ or $\langle\mathrm{M}, \Sigma\rangle=\mid \alpha$, all that is required is to establish that where $\langle\mathrm{M}, \Sigma\rangle$ and $\left\langle\mathrm{M}^{\prime}, \Sigma^{\prime}\right\rangle$ are any models $\langle\mathrm{M}, \Sigma\rangle \mid=\alpha$ iff $\left\langle\mathrm{M}^{\prime}, \Sigma^{\prime}\right\rangle \mid=\alpha$. The proof is by induction on the construction of $\alpha$. The only atomic case is $\perp$, and $\langle\mathrm{M}, \Sigma\rangle=\perp$, for every $\langle\mathrm{M}, \Sigma\rangle$, and so $\langle\mathrm{M}, \Sigma\rangle \mid=\perp$ iff $\left\langle\mathrm{M}^{\prime}, \Sigma^{\prime}\right\rangle|=\alpha .\langle\mathrm{M}, \Sigma\rangle|=\alpha \supset \beta$ iff either $\langle\mathrm{M}, \Sigma\rangle$ $\Rightarrow \alpha$ or $\langle\mathrm{M}, \Sigma\rangle \mid=\beta$, iff (by the induction hypothesis) either $\left\langle\mathrm{M}^{\prime}, \Sigma^{\prime}\right\rangle \Rightarrow \alpha$ or $\left\langle\mathrm{M}^{\prime}, \Sigma^{\prime}\right\rangle \mid=\beta$, iff $\left\langle\mathrm{M}^{\prime}, \Sigma^{\prime}\right\rangle|=\alpha \supset \beta .\langle\mathrm{M}, \Sigma\rangle|=\square \alpha$ iff $\langle\mathrm{M}, \Sigma\rangle \mid=s(\alpha)$ for every $s \in$ $\Sigma$. Since $\alpha$ is constant $s(\alpha)$ is $\alpha$, and, by the induction hypothesis, $\langle\mathrm{M}, \Sigma\rangle \mid=$ $\alpha$ iff $\left\langle\mathrm{M}^{\prime}, \Sigma^{\prime}\right\rangle \mid=\alpha$.

Call $s$ a final substitution iff

$$
\text { For every variable } p \text { either } s(p)=\top \text { or } s(p)=\perp \text {. }
$$

Call $\langle\mathrm{M}, \Sigma\rangle$ a final model iff $\Sigma$ satisfies Ref and Trans, and contains a final substitution. For soundness we prove that $\mathbf{M}$ is valid in every final McKinsey model $\langle\mathrm{M}, \Sigma\rangle$. As noted in p. 70 of Cresswell $2013 \mathbf{M}$ is trivially equivalent to:

$$
\text { M1 } \diamond(\diamond p \supset \square p)
$$

and so it is sufficient to prove that $\mathrm{M} 1$ is $\langle\mathrm{M}, \Sigma\rangle$-valid. That is to say

THEOREM 8 For any wff $\alpha$ and any final model $\langle\mathrm{M}, \Sigma\rangle, \diamond(\diamond \alpha \supset \square \alpha)$ is $\langle\mathrm{M}, \Sigma\rangle$ true.

Proof. Let $\langle\mathrm{M}, \Sigma\rangle$ be any final model. Then there will be a final $s \in \Sigma$, and in that case,by lemma $7 s(\diamond \alpha \supset \square \alpha)$ will be equivalent to either $\top \supset \top$ or $\perp \supset$ $\perp$, and in either case $\langle\mathrm{M}, \Sigma\rangle \mid=s(\diamond \alpha \supset \square \alpha)$, and so $\langle\mathrm{M}, \Sigma\rangle \mid=\diamond(\diamond \alpha \supset \square \alpha)$. 
For completeness we produce, for any final Kripke model, a particular final McKinsey model; and shew that the same formulae satisfy it as satisfy the Kripke model. We assume that our system $S$ is S4M, and that we have a final S4M model $\langle\mathrm{W}, \mathrm{R}, \mathrm{V}\rangle$ with a set $\mathrm{F}_{\mathrm{R}}$ defined as in section 2 . To take account of the finality condition we add a set $\mathrm{F}^{*}$ of final substitutions defined so that $s$ $\in \mathrm{F}^{*}$ iff for every non-final $w \in \mathrm{W}$ and every final $w^{\prime} \in \mathrm{W}$, such that $w \mathrm{R} w{ }^{\prime}$ :

$$
\begin{aligned}
& \text { For every } p \in \mathrm{P}, s\left(p_{\mathrm{w}}\right)=\top \text { if } \mathrm{V}\left(p, w^{\prime}\right)=1 \text { and } s\left(p_{\mathrm{w}}\right)=\perp \text { if } \\
& \mathrm{V}\left(p, w^{\prime}\right)=0 .
\end{aligned}
$$

Notice that, since Kripke models for S4M are based on transitive frames, if $w_{1}$ can see a final world $w_{2}$, and if $w_{3}$ can see $w_{1}$ then $w_{3}$ can also see $w_{2}$. This ensures that if in $\mathrm{F}^{*}$ there is some $s$ which satisfies (23) and for some $w_{3}, s\left(p_{w 3}\right)=p_{w 2}$ then there is already some $s^{\prime} \in \mathrm{F}^{*}$ such that $s^{\prime}\left(p_{w 3}\right)$ satisfies

$$
\begin{aligned}
& \text { For every } p \in \mathrm{P}, s^{\prime}\left(p_{\mathrm{w}}\right)=\top \text { if } \mathrm{V}\left(p, w^{\prime}\right)=1 \text { and } s^{\prime}\left(p_{\mathrm{w}}\right)=\perp \text { if } \\
& \mathrm{V}\left(p, w^{\prime}\right)=0 .
\end{aligned}
$$

In defining $\langle\mathrm{M}, \Sigma\rangle$ we now require that $\mathrm{F}^{*} \subseteq \Sigma$. Using $s_{\mathrm{f}}$ as defined in (7) we have, in place of (8), that $\Sigma$ is

$$
\left\{s: \exists f\left(f_{\mathrm{R}} \wedge s=s_{\mathrm{f}}\right)\right\} \cup F *
$$

$\alpha_{w}$ (in $\mathcal{L}_{\omega}$ ) has also to be defined a little differently from (11).

(i) For non-final $w, \alpha_{w}$ is $\alpha$ with $p_{w}$ replacing $p$ for every variable $p$

(ii) For final $w, \alpha_{w}$ is $\alpha$ with $\top$ replacing $p$ if $\mathrm{V}(p, w)=1$, and $\perp$ replacing $p$ if $V(p, w)=0$.

At this point there is a problem of labelling. Where $w$ is a non-final world, any variable $p$ in $\alpha$ becomes $p_{\mathrm{w}}$ in $\alpha_{\mathrm{w}}$. But where $w$ is final then it will be either $\top$ or $\perp$, and will not be $p_{\mathrm{w}}$. To indicate this where there may be a danger of confusion I shall use the notation $\alpha_{\mathrm{w}}{ }^{\mathrm{c}}$ to indicate the constant wff formed by replacing $p$ by $\top$ or $\perp$ according as $\mathrm{V}(p, w)=1$ or $\mathrm{V}(p, w)=0$. That is to say, where $w$ is a final world $\alpha_{w}$ is $\alpha_{\mathrm{w}}{ }^{\mathrm{c}}$. The assignment to variables is as in (15). We have to prove an analogue of theorem 1. First a lemma:

Lemma 9 If $w$ is a final world in $\langle\mathrm{W}, \mathrm{R}, \mathrm{V}\rangle$ and $\langle\mathrm{M}, \Sigma\rangle$ is constructed from $\langle\mathrm{W}, \mathrm{R}, \mathrm{V}\rangle$ in accordance with 15 and 25 then $\langle\mathrm{M}, \Sigma\rangle \mid=\alpha_{\mathrm{w}}{ }^{\mathrm{c}}$ iff $\mathrm{V}(\alpha, w)=1$.

Proof. The proof is by induction on the construction of $\alpha$. Observe first that since $w$ is final then, for every $\mathrm{wff} \beta, \mathrm{V}(\square \beta, w)=\mathrm{V}(\beta, w)$. For any $p \in \mathrm{P}, p_{\mathrm{w}}{ }^{\mathrm{c}}$ is 
defined so that the lemma holds. It holds trivially for $\perp$ and is preserved by $\supset$, given that $(\beta \supset \gamma)_{w}{ }_{w}^{\mathrm{c}}$ is $\left(\beta_{\mathrm{w}}{ }^{\mathrm{c}} \supset \gamma_{w}{ }^{\mathrm{c}}\right)$. For $\square$, given that $(\square \alpha)_{\mathrm{w}}{ }^{\mathrm{c}}$ is $\square \alpha_{\mathrm{w}}{ }_{\mathrm{w}}^{\mathrm{c}}$, if $\langle\mathrm{M}, \Sigma\rangle=\square \alpha_{\mathrm{w}}{ }^{\mathrm{c}}$ then, for some $s \in \Sigma,\langle\mathrm{M}, \Sigma\rangle=\mid s\left(\alpha_{\mathrm{w}}{ }^{\mathrm{c}}\right)$. But $\alpha_{\mathrm{w}}{ }^{\mathrm{c}}$ is a constant wff, and so, by lemma $7,\langle\mathrm{M}, \Sigma\rangle=\alpha_{\mathrm{w}}{ }^{\mathrm{c}}$, and so, by the induction hypothesis, $\mathrm{V}(\alpha, w)=0$, and so $\mathrm{V}(\square \alpha, w)=0$. Conversely, if $\mathrm{V}(\square \alpha, w)=0$ then, since $w$ is final, $\mathrm{V}(\alpha, w)=0$, and so $\langle\mathrm{M}, \Sigma\rangle \Rightarrow \square \alpha_{\mathrm{w}}{ }^{\mathrm{c}}$.

THEOREM 10 For any wff $\alpha$ in $\mathrm{L},\langle\mathrm{M}, \Sigma\rangle \mid=\alpha_{w}$ iff $\mathrm{V}(\alpha, w)=1$.

Proof. Given lemma 9 we may assume the theorem for final $w$, since in that case $\alpha_{w}$ is $\alpha_{\mathrm{w}}{ }^{\mathrm{c}}$. So suppose that $w$ is non-final. The result is defined to hold for the variables, and clearly holds for $\perp$ and is preserved by $\supset$. For $\square$, suppose first that $\mathrm{V}(\square \alpha, w)=0$. Then there is some $w^{\prime}$ (which may be $w)$ such that $w \mathrm{R} w^{\prime}$ and $\mathrm{V}\left(\alpha, w^{\prime}\right)=0$. So by the induction hypothesis or by lemma $9\langle\mathrm{M}, \Sigma\rangle=\mid \alpha_{w^{\prime}}$. So by 13, there is some $s \in \Sigma$ such that $s\left(\alpha_{w}\right)=\alpha_{w^{\prime}}$, and therefore $\langle\mathrm{M}, \Sigma\rangle=\square \alpha_{w}$, i.e., $\langle\mathrm{M}, \Sigma\rangle=(\square \alpha)_{w}$. Now suppose that $\langle\mathrm{M}, \Sigma\rangle$ $=(\square \alpha)_{w}$. Then $\langle\mathrm{M}, \Sigma\rangle \Rightarrow \square \alpha_{w}$, and so for some $s \in \Sigma,\langle\mathrm{M}, \Sigma\rangle=s\left(\alpha_{w}\right)$. Now $s\left(\alpha_{w}\right)$ is $\alpha_{w^{\prime}}$ for some $w^{\prime}$, and so, by the induction hypothesis or lemma 9, $\mathrm{V}\left(\alpha, w^{\prime}\right)=0$, and since, by $14, w \operatorname{R} w^{\prime}$ then $\mathrm{V}(\square \alpha, w)=0$.

\section{$6 \quad$ Full models}

Although what we have proved goes some way towards articulating McKinsey's motivating idea it does not go all the way. One tempting way of articulating this idea is set out on p. 68 of Cresswell 2013:

$\langle\mathrm{M}, \Sigma\rangle \mid=\square \alpha$ iff $\langle\mathrm{M}, \Sigma\rangle \mid=\alpha^{\prime}$ for every wff $\alpha^{\prime}$ which can be obtained from $\alpha$ by uniform substitution for the propositional variables of $\alpha$.

Although (27) seems natural, it does, as noted in that article, have some problems. One of these is that it appears to allow the substitution of modal wff for the propositional variables, which leads to problems of the kind mentioned in section 1 of the present paper, since it can result in cases where, in order to evaluate the truth of $\square \alpha$ you might already have to know its truth value. Of course modal wff can simply be ruled out by fiat as done in section 1. More importantly, not only does (27) permit complex PC-wff to be substituted for variables - it seems to require that every PC-wff is eligible. That is to say it requires a model $\langle\mathrm{M}, \Sigma\rangle$ to be full in the sense of requiring that $\Sigma$ contain every substitution which takes variables into PCwff. What this means is that $\sim \square p$ is $\langle\mathrm{M}, \Sigma\rangle$-true in every McKinsey model. 
This is because in a full model there is some $s \in \Sigma$ such that $\langle\mathrm{M}, \Sigma\rangle \Rightarrow s(\perp)$, and so, in every full model, $\langle\mathrm{M}, \Sigma\rangle \Rightarrow \square p$, so that $\langle\mathrm{M}, \Sigma\rangle \mid=\sim \square p$, which is not a theorem of S4M. In itself this may not be a problem, since, although $\sim \square p$ may be true in every full McKinsey model, it is not valid in every full model since $\sim \square \top$ is false in every McKinsey model, and for that reason $\sim \square p$ has a false instance in every model, and so is not valid. ${ }^{13}$ Full models are certainly final, and therefore their logic, whether axiomatisable or not, contains S4M. One might therefore be tempted to conjecture that S4M is characterised by the class of full McKinsey models. I have no proof that this conjecture is true or that it is false.

\section{$7 \quad$ Appendix ${ }^{14}$}

While I believe that the paper is faithful to McKinsey's intentions there are certainly differences between the presentation offered here and McKinsey's own. The purpose of this Appendix is to shew how the results established here apply to McKinsey's own presentation. To that end I will shew how to obtain what I have called a 'McKinsey model' from McKinsey's own exposition.

McKinsey first introduces a language $L$ of 'sentences' whose nature is unspecified except that it contains the symbols $\sim$ and '. '. (It could equally have been $\supset$ and $\perp$.) He then assumes a subset $T$ of $L$ which is understood to be the set of 'true' sentences of $L$. $\sim$ and . are understood as logical constants which have a fixed interpretation. Adapting $T$ to a language based on $\supset$ and $\perp$ we can say that $T$ is so constrained that $\perp \notin T$, and $\alpha \supset \beta \in T$ iff either $\alpha \notin T$ or $\beta \in T$. The difference between McKinsey's way of proceeding, and that of the present paper is that McKinsey seems to feel that $L$ and $T$ are fixed, and that, in particular, $T$ is not provided by a model. This may be because he would feel that any reference to a model could compromise the 'syntactic' emphasis of his work.

Even when he extends $L$ to $L_{1}$ by the addition of the modal operator $\diamond$ (in our case $\square$ ) McKinsey reminds us on p. 85 that his system contains 'only constant sentences', and that the modal logic it leads to is not any of the usual ones. To obtain a modal logic of the usual sort McKinsey moves

\footnotetext{
${ }^{13}$ Unlike truth in a model, where $\sim \alpha$ is true iff $\alpha$ is false it is not the case that $\sim \alpha$ is valid in the model just because $\alpha$ is not valid in that model. So we cannot conclude that $\sim p$ is valid just because $\square p$. is not.

${ }^{14}$ I am grateful to a referee for this journal for reminding me of the importance of the difference between the way of proceeding in the bulk of this paper and McKinsey's. Although they turn out to be equivalent, this requires to be proved.
}

Australasian Journal of Logic (17:2) 2020, Article no. 3 
to a language $L_{2}$ (in effect our $\mathcal{L}$ ) which is like $L_{1}$ except that its formulae are made up of propositional variables. A formula $\alpha\left(p_{1}, \ldots, p_{\mathrm{n}}\right)$ is in $T_{2}$ iff $\alpha\left(\alpha_{1}, \ldots, \alpha_{\mathrm{n}}\right)$ is in $T_{1}$ for all $\alpha_{1}, \ldots, \alpha_{\mathrm{n}}$ where $\alpha_{1}, \ldots, \alpha_{\mathrm{n}}$ are sentences of $L_{1}$. The aim is to shew that where $\langle\mathrm{M}, \Sigma\rangle$ is a McKinsey model as in $\S 1$ above we can define a translation $\pi_{\langle M, \Sigma\rangle}$ which takes sentences from the fixed $\left\langle L_{1}, T_{1}\right\rangle$ and translates them into formulae of $\mathcal{L}$ which are true in $\langle\mathrm{M}, \Sigma\rangle$ iff the originals are in $T_{1}$.

Since both $L_{1}$ and $\mathcal{L}$ are denumerable languages we may assume that the variables of $\mathcal{L}$ may be put into 1-1 correspondence with the nonlogical sentences of $L_{1}$ (i.e., sentences without any occurrence of $\perp, \supset$ or $\square$ ). For any such sentence $\alpha$ let $p_{\alpha}$ be the propositional variable in $\mathcal{L}$ associated with $\alpha$. Where $\langle\mathrm{M}, \Sigma\rangle$ is a McKinsey model as defined in $\S 1$ above let $\pi\langle M, \Sigma\rangle$ be a 1-1 function from the non-logical sentences of $L_{1}$ to the propositional variables of $\mathcal{L}$ or their negations, satisfying the condition that (i) If $\alpha \in T_{1}$, then $\pi_{\langle M, \Sigma\rangle}(\alpha)$ is $p_{\alpha}$ if $\langle\mathrm{M}, \Sigma\rangle \mid=p_{\alpha}$ and $\pi_{\langle M, \Sigma\rangle}(\alpha)$ is $\sim p_{\alpha}$ if $\langle\mathrm{M}, \Sigma\rangle=\mid p_{\alpha}$ and (ii) if $\alpha \notin T_{1}$, then $\pi_{\langle M, \Sigma\rangle}(\alpha)$ is $\sim p_{\alpha}$ if $\langle\mathrm{M}, \Sigma\rangle \mid=p_{\alpha}$ and $\pi_{\langle M, \Sigma\rangle}(\alpha)$ is $p_{\alpha}$ if $\langle\mathrm{M}, \Sigma\rangle$ $=p_{\alpha}$. It is clear that for every $\langle\mathrm{M}, \Sigma\rangle$ there is some $\pi_{\langle M, \Sigma\rangle}(\alpha)$ which satisfies the condition. $\pi_{\langle M, \Sigma\rangle}$ can then be extended to all wff of $L_{1}$ by requiring that for any $\alpha$ and $\beta$ in $L_{1}, \pi_{\langle M, \Sigma\rangle}(\perp)=\perp, \pi_{\langle M, \Sigma\rangle}(\alpha \supset \beta)=\left(\pi_{\langle M, \Sigma\rangle}(\alpha) \supset \pi_{\langle M, \Sigma\rangle}(\beta)\right)$, and $\pi_{\langle M, \Sigma\rangle}(\square(\alpha))=\square \pi_{\langle M, \Sigma\rangle}(\alpha)$. Since $\Sigma$ is a set of substitutions relative to $\mathcal{L}$ we need to specify an equivalent set $S_{\Sigma}$ of substitutions in $L_{1}$, in which for every non-logical sentence $\alpha$ of $L_{1} s(\alpha)$ is a sentences of $L_{1}$ which does not contain $\square$. Let $S_{\Sigma}$ be the set of all all substitutions in $L_{1}$ which correspond with some $s \in \Sigma$ in the sense that for every variable $p_{\alpha}, s\left(p_{\alpha}\right)=\pi_{\langle M, \Sigma\rangle}\left(s_{\Sigma}(\alpha)\right)$.

LEMMA 12 For every sentence $\alpha$ of $L_{1},\langle\mathrm{M}, \Sigma\rangle \mid=\pi_{\langle M, \Sigma\rangle}(\alpha)$ iff $\alpha \in T_{1}$.

The proof is by induction on the construction of $\alpha$. The lemma clearly holds for $\perp$. Consider a sentence of $L_{1}$ without logical constants. There are two cases to consider. (i) If $\alpha \in T_{1}$, then $\pi_{\langle M, \Sigma\rangle}(\alpha)$ is $p_{\alpha}$ if $\langle\mathrm{M}, \Sigma\rangle \mid=p_{\alpha}$ and $\pi_{\langle M, \Sigma\rangle}(\alpha)$ is $\sim p_{\alpha}$ if $\langle\mathrm{M}, \Sigma\rangle=p_{\alpha}$. So in this case $\langle\mathrm{M}, \Sigma\rangle \mid=\pi_{\langle M, \Sigma\rangle}(\alpha)$ iff $\alpha \in T_{1}$. (ii) If $\alpha \notin T_{1}$, then $\pi_{\langle M, \Sigma\rangle}(\alpha)$ is $\sim p_{\alpha}$ if $\langle\mathrm{M}, \Sigma\rangle \mid=p_{\alpha}$ and $\pi_{\langle M, \Sigma\rangle}(\alpha)$ is $p_{\alpha}$ if $\langle\mathrm{M}, \Sigma\rangle=p_{\alpha}$, so in this case also $\langle\mathrm{M}, \Sigma\rangle \mid=\pi_{\langle M, \Sigma\rangle}(\alpha)$ iff $\alpha \in T_{1}$. For $\supset, \pi_{\langle M, \Sigma\rangle}(\alpha \supset \beta)=\left(\pi_{\langle M, \Sigma\rangle}(\alpha)\right.$ $\left.\supset \pi_{\langle M, \Sigma\rangle}(\beta)\right)$, and $\langle\mathrm{M}, \Sigma\rangle \mid=\left(\pi_{\langle M, \Sigma\rangle}(\alpha) \supset \pi_{\langle M, \Sigma\rangle}(\beta)\right.$ iff $\langle\mathrm{M}, \Sigma\rangle=\left(\pi_{\langle M, \Sigma\rangle}(\alpha)\right.$ or $\langle\mathrm{M}, \Sigma\rangle \mid=\pi_{\langle M, \Sigma\rangle}(\beta)$ iff $\alpha \notin T_{1}$ or $\beta$ is in $T_{1}$. For $\square$ we note that since $\pi_{\langle M, \Sigma\rangle}(\square \alpha)$ is $\square \pi_{\langle M, \Sigma\rangle}(\alpha)$ then it is always of higher degree than $\pi_{\langle M, \Sigma\rangle}(\alpha)$ and so we may assume that the result has already been established not only for $\alpha$ itself but for any substitution instance of $\alpha$, because substitutions only involve the substitution of modal-free wff for the non-logical formulae. So suppose that $\langle\mathrm{M}, \Sigma\rangle=\square \alpha$. Then for some $s \in \Sigma,\langle\mathrm{M}, \Sigma\rangle=s\left(\pi_{\langle M, \Sigma\rangle}(\alpha)\right)$, and therefore 
where $s_{\Sigma} \in \mathrm{S}_{\Sigma}$ corresponds with $\left.s,\langle\mathrm{M}, \Sigma\rangle=\pi_{\langle M, \Sigma\rangle}\left(s_{\Sigma}(\alpha)\right)\right)$, and so $\alpha \notin T_{1}$, and then $\square \alpha \notin T_{1}$. Similarly, if $\square \alpha \notin T_{1}$ then for some $\left.s_{\Sigma} \in \mathrm{S}_{\Sigma}, s_{\Sigma}(\alpha)\right) \notin T_{1}$, and therefore, where $s$ corresponds to $s_{\Sigma},\langle\mathrm{M}, \Sigma\rangle=s\left(\pi_{\langle M, \Sigma\rangle}(\alpha)\right)$, and so $\langle\mathrm{M}, \Sigma\rangle=$ $\square \pi_{\langle M, \Sigma\rangle}(\alpha)$, i.e. $\langle\mathrm{M}, \Sigma\rangle=\pi_{\langle M, \Sigma\rangle}(\square \alpha)$.

\section{Conclusion}

One lesson which emerges from this work is an appreciation of the superiority of the current possible worlds semantics based on frames and models, both in terms of an intuitive understanding of modality, and also in terms of the ease of working with particular systems. In the final section of his paper McKinsey hopes to deal with semantic issues 'in a later paper' (p. 94) which, as far as I am aware was never written. It would be interesting to know whether he would have thought that the use of 'possible worlds' in current semantics compromises the aim of producing a 'sytactical' account of necessity. ${ }^{15}$

REFERENCES

Carnap, R, 1946, Modalities and quantification. The Journal of Symbolic Logic, Vol 11, pp. 33-64

Cresswell, M.J., 2013, Carnap and McKinsey: Topics in the pre-history of possible worlds semantics Proceedings of the 12th Asian Logic Conference, J. Brendle, R. Downey, R. Goldblatt and B. Kim (eds), World Scientific, 2013, pp 53-75.

Drake, F.R., 1962, On McKinsey's syntactical characterizations of systems of modal logic. The Journal of Symbolic Logic, Vol 27, pp. 400-406.

Goldblatt, R.I., 2006, Mathematical Modal Logic: A View of its Evolution. Logic and the Modalities in the Twentieth Century, Handbook of the History of Logic, vol. 7, Dov M. Gabbay, John Woods, Eds, Elsevier, 2006, pp. 1-98.

Hughes, G.E. and M.J. Cresswell, 1984, A Companion to Modal Logic, London, Methuen, 1996, A New Introduction to Modal Logic, London, Routledge

Humberstone, I.L., 2015, Philosophical Applications of Modal Logic, London, College Publications

\footnotetext{
${ }^{15}$ I am grateful to an anonymous referee for an extremely thorough and supererogatory report on an earlier version of the paper, which revealed numerous infelicities and some downright errors. More importantly it offered invaluable suggestions for overcoming them.
} 
Lemmon, E.J., and D.S. Scott, 1977, The 'Lemmon Notes': An Introduction to Modal Logic, ed. K. Segerberg, Oxford, Basil Blackwell

Lewis, C.I., and C.H. Langford, 1932, Symbolic Logic, New York, Dover publications

McKinsey, J.C.C., 1945, On the syntactical construction of systems of modal logic. The Journal of Symbolic Logic, Vol 10, pp. 83-94.

Makinson, D.C., 1966, On some completeness theorems in modal logic, Zeitschrift für mathematische Logik und Grundlagen der Mathematik, Vol 12, pp. 379-84

Quine, W.V.O, 1934, Ontological remarks on the propositional calculus. Mind, Vol 433, pp. 473-476

Sobociński, B., 1964, Remarks about the axiomatizations of certain modal systems, Notre Dame Journal of Formal Logic, Vol 5, pp 71-80

Whitehead, A.N., and B.A.W. Russell, 1910, Principia mathematica, Cambridge, Cambridge University Press, 3 vols., First edition 1910-1913, Second edition 1923-1927 